\title{
IoT-Assisted Hybrid Intelligent Learning Architecture Based on Digital Education in a Diverse Society
}

\author{
Zongpei Ma \\ Zhengzhou Normal University, Zhengzhou 450044, China \\ Correspondence should be addressed to Zongpei Ma; mazongpei@zznu.edu.cn
}

Received 26 September 2021; Revised 23 October 2021; Accepted 30 October 2021; Published 24 November 2021

Academic Editor: Hai Dong

Copyright $\odot 2021$ Zongpei Ma. This is an open access article distributed under the Creative Commons Attribution License, which permits unrestricted use, distribution, and reproduction in any medium, provided the original work is properly cited.

\begin{abstract}
The existing research paradigm has caused the research of educational theory to be criticized for lack of logic and scientificity. With the gradual deepening and refinement of the development of smart education platforms, the educational process can be quantified, and the research paradigm of educational science has moved from a sampling model to a full-sample model, and it has become a real empirical science. This paper combines the Internet of Things technology to construct a hybrid intelligent learning system and applies it to digital education in a diverse society to construct a functional structure of the extraction system. Moreover, starting from the actual situation of intelligent education, this paper combines a simulation system to implement an IoT-assisted hybrid intelligent learning architecture and evaluates the teaching effect of the system. Through experimental research, it can be known that the IoT-assisted hybrid intelligent learning framework based on social diversified digital education has certain effects and has a certain reference effect for the development of subsequent intelligent education.
\end{abstract}

\section{Introduction}

The Internet of Things is the third wave in the development of the world's information industry. The Internet of Things, in layman's terms, is a network that connects objects to objects based on the Internet. It uses sensors, positioning systems, and other information equipment to connect any object to the Internet according to a certain protocol and can realize various information exchange and communication between interconnected objects and finally realize the intelligent management of objects [1]. The realization of the overall concept of the Internet of Things relies on a number of technological inventions, and its key technologies cover the entire information processing process from acquisition, transmission, processing, and storage to application. For objects connected to the network, the Internet of Things has a perception function. By reading the electronic tag of the object, various information can be collected in real time, and after a series of processing, the data information is transmitted to the Internet according to the communication protocol [2]. Finally, it uses intelligent computing technology to process the collected data and information and finally realizes the intelligent control of networked objects. Generally speaking, the network architecture of the Internet of Things is a three-layer structure, namely, the perception layer, the network layer, and the application layer. The perception layer is the source of the Internet of Things information and the bottom layer of the Internet of Things architecture. At this layer, it is mainly responsible for collecting and perceiving various raw data information. The network layer is responsible for connecting various data and information collection and perception networks to the Internet, so as to realize the storage, integration, and longdistance transmission of information. The application layer is responsible for data mining, that is, the discovery and presentation of various services, and decision-making on applications. The following will introduce the core technology of the Internet of Things system RFID radio frequency identification technology and WSN wireless sensor network and Internet of Things grid technology [3].

The smart education platform gathers countless data that was previously unseen, uncollected, and ignored. Whether it is the reform of the macro system and system, or the reform of the micro teaching methods and management methods, it 
can be through the in-depth mining of the smart education platform and the association with big data in other fields (such as public security, transportation, social security, and medical care). Analyze and find the crux of the problem, identify the unique laws of educational development in different regions, and then prescribe the right medicine to implement reforms. Therefore, educational decision-making will no longer rely excessively on experience, brainstorming, and simple statistical results but will turn to scientific decision-making based on data. In the learning environment of the smart education platform, techniques and methods of mathematical statistics, machine learning, and data mining can be used to process and analyze data, discover the mechanism of student learning, and use it to optimize learning and develop adaptive learning, self-directed learning; it can also analyze the teacher's course teaching in depth, so that teachers can provide students with more targeted teaching interventions based on data analysis; it can also evaluate the management effectiveness of managers in order to improve the existing assessment methods and management methods. The smart education platform can continuously collect learners' learning behavior data and perform intelligent analysis, such as pushing appropriate learning resources based on learner models, conducting personalized learning evaluations, providing accurate diagnosis results, and providing them with truly personalized learning resources, learning activities, learning paths, learning tools and services, etc. In the smart education platform environment, various data collection technologies can be used to collect information on school profiles, faculty, school funding, books and materials, equipment, majors and courses, teaching management, teaching process, teaching results, basic student conditions, etc. In order to achieve the purpose of comprehensive monitoring of education quality, a comprehensive and dynamic teaching quality monitoring system is formed.

This article combines the Internet of Things technology to construct a hybrid intelligent learning system, applies it to digital education in a diverse society, and verifies the system to provide a theoretical reference for subsequent intelligent education.

\section{Related Work}

The Internet of Things, literally understood, is the Internet between things and things [4]. The concept of the Internet of Things was first proposed in 1999 by Professor Kevin Ashton of the Massachusetts Institute of Technology in the United States. It is based on Internet technology to use radio frequency identification, wireless data communication, and other technologies to construct a network covering everything in the world, so as to realize the automatic identification of items and the interconnection and sharing of information [5]. The US “Technology Review" believes that the sensor network technology in the Internet of Things technology will become one of the top ten technologies that will change the world in the future.

After the construction plan of the smart education platform was proposed, many experts and scholars, government education departments, and enterprises have begun to conduct various researches, analyses, and constructions on the theoretical basis, development plan, framework scheme, and design ideas of the smart education platform. In terms of the theoretical construction of the smart education platform, many experts and scholars start with the concept of smart education as the core and start with the current education status and educational needs to study the theoretical design and development of the smart education platform [6]. Literature [7] proposed the following ideas for the construction of a comprehensive service platform for regional smart education. The overall plan of the platform is coordinated and implemented step by step and then driven by user needs to integrate various technologies for innovation. After that, the platform is gradually upgraded and gradually improved, and then it is interconnected with the national education resources and education management platform to open up and expand. Literature [8] proposed that the construction of a smart education platform must be innovative in three aspects: technological innovation (intelligence of terminals, systems and platforms), model innovation (mixed education becomes the mainstream of platform teaching), and evaluation innovation (reform traditional "input" education). Literature [9] proposed that the design of smart education platform should follow six principles: advanced and scientific principles, comprehensive and integrated principles, ease of operation, scalability, and security. Literature [10] proposes to build a regional smart education platform to achieve the use of cloud computing concepts, the integration of educational information hubs, and the sharing of educational information resources. Literature [11] proposed that the smart education cloud computing platform must have five basic characteristics, namely, on-demand self-service, ubiquitous network access, division of independent resource pools, rapid flexibility, and measurable services. The key technologies of the smart education cloud platform need to include five aspects: service scenario recognition, smart information extraction, smart information processing, smart information retrieval, and smart information push. In terms of the construction and development of smart education platforms, major IT companies and enterprises around the world rely on their own strong economic and technical strength to continue to cooperate with governments and colleges and universities around the world to develop smart education platforms. Taking IBM as an example, in terms of smart education, IBM theoretically put forward five major strategies for learning with any device, shifting to a learner-centered, building a learning community, professional learning services, and systematic education [12]. At the same time, IBM relied on its strong economic and technical strength to cooperate with many top universities at home and abroad to create a "smart campus" and summed up many effective practical experience, such as integrating campus information platform, helping campus transformation, modular, highperformance computing resource services [13].

\section{Internet of Things Learning Method}

Internet of Things clustering is an unsupervised classification process. It does not need to provide prior knowledge about sample category labels. It can only give a reasonable division of things based on the similarity between samples, 
which is different from the classification in data mining. Clustering pays more attention to finding the potential structure of the sample set and divides the set into several subsets with a smaller degree of relevance. From the point of view of the classification of sample points, clustering methods are mainly divided into two categories: the first category is hard partitioning, which stipulates that each sample belongs to only a certain category, and the boundary between the categories is clear: the second category is a softening score, that is, fuzzy clustering. It no longer requires that each sample point belong to only one class but uses the degree of membership to express the probability that the sample belongs to a certain class. The greater the degree of membership, the more likely the sample point belonging to this category. Since natural processes are generally fuzzy, fuzzy clustering is closer to the real situation in nature. The focus of clustering technology research mainly includes cluster analysis, similarity measurement, and cluster validity evaluation.

The mathematical model of cluster analysis is as follows: we set $X=\left\{x_{1}, x_{2}, \ldots, x_{n}\right\}$ as the sample set to be clustered, which includes $n$ sample points. We denote each sample in $X$ as $x_{i}=\left\{x_{i 1}, x_{i 2}, \ldots, x_{i m}\right\}$, which has $m$ feature attributes. Cluster analysis is to divide the $n$ samples in the sample set $X$ into $k$ disjoint sample subsets $C_{1}, C_{2}, \ldots, C_{k}$ according to their characteristic attributes and require them to meet the following conditions [14]:

$$
C_{1} \cup C_{2} \cup \ldots \cup C_{K}=C ; C_{i} \cap C_{j}=\varnothing, 1 \leq i \neq j \leq k .
$$

The membership relationship of sample $x_{j}(1 \leq j \leq n)$ relative to subset $C_{i}(1<i \leq k)$ can be expressed as a membership function [15]:

$$
u_{C_{i}}\left(x_{j}\right)=u_{i j}=\left\{\begin{array}{l}
1, x_{j} \in C_{i} \\
0, x_{j} \notin C_{i}
\end{array} .\right.
$$

Among them, each sample can and can only belong to a certain subset, and each subset is nonempty, so the membership function must satisfy the condition $u_{i j} \in M_{h k}$.

$$
M_{h k}=\left\{u_{i k} \mid u_{i k} \in\{0,1\} ; \sum_{i=1}^{k} u_{i j}=1 ; 0<\sum_{j=1}^{n} u_{i j}<n, \forall i\right\} .
$$

In fuzzy clustering, the sample set and $X$ are divided into $k$ fuzzy subsets $\widetilde{C}_{1}, \widetilde{C}_{2}, \ldots, \widetilde{C}_{k}$, and the membership degree $u_{i j} \in[0,1]$ of the sample; the conditions that need to be met are as follows:

$$
\underset{i=1}{K} \operatorname{supp}\left(\widetilde{C}_{i}\right)=C ; u_{i j} \in[0,1] ; \sum_{i=1}^{k} u_{i j}=1 ; 0<\sum_{j=1}^{n} u_{i j}<n, \forall i .
$$

Among them, supp represents the support set of the fuzzy set.

Similarity measurement is an important problem in clustering. It is the basis for judging the similarity between different samples. Therefore, choosing a suitable similarity measurement method can greatly improve the accuracy of the clustering algorithm. There are many ways to measure similarity in clustering, and the commonly used methods are as follows:

We have two sample points $x_{1}=\left(x_{11}, x_{12}, \ldots, x_{1 m}\right)$ and $x_{2}=\left(x_{21}, x_{22}, \ldots, x_{2 m}\right)$, the distance between them is $d_{1,2}$, and the commonly used distance in cluster similarity measurement is expressed as follows:

(a) Euclidean distance is [16]

$$
d_{1,2}=\sqrt{\sum_{j=1}^{m}\left(x_{i, j}-x_{2, j}\right)^{2}} .
$$

(b) Manhattan distance is

$$
d_{1,2}=\sum_{j=1}^{m}\left|x_{i, j}-x_{2, j}\right|
$$

(c) Chebyshev distance is

$$
d_{1,2}=\lim _{q \longrightarrow \infty}\left(\sum_{j=1}^{m}\left|x_{i, j}-x_{2, j}\right|^{q}\right)^{1 / q} .
$$

(d) Minkowski distance is

$$
d_{1,2}=\sqrt[p]{\sum_{j=1}^{m}\left|x_{i, j}-x_{2, j}\right|^{p}}
$$

Among them, $p$ is a variable parameter. When $p=1$, the Minkowski distance is the Manhattan distance: when $p=2$, it is the Euclidean distance; when $p->\infty$, it is the Chebyshev distance. According to the different parameters, Min's distance can represent a certain kind of distance [17].

(e) Mahalanobis distance is as follows:

We have sample sets $X=\left\{x_{1}, x_{2}, \ldots, x_{n}\right\}$ and $x_{i}=\left\{x_{i 1}, x_{i 2}, \ldots, x_{i m}\right\}$, the covariance matrix is $S$, and the mean value is denoted as vector $u$. Then, the Mahalanobis distance of the sample vector $X$ to $u$ is expressed as

$$
D(X)=\sqrt{(X-\mu)^{T} S^{-1}\left(X_{i}-\mu\right)} .
$$

Among them, the Mahalanobis distance between the vectors $X i$ and $j$ is defined as

$$
D\left(X_{i}, X_{j}\right)=\sqrt{\left(X_{i}-X_{j}\right)^{T} S^{-1}\left(X_{i}-X_{j}\right)} .
$$

When the covariance matrix is the identity matrix, the Mahalanobis distance is expressed as Euclidean distance.

(f) Hamming distance is as follows:

Hamming distance is a relatively special distance measurement method, which is a distance 
measurement method based on binary codes. We suppose that $s 1$ and $s 2$ are two equal-length binary code strings, and the Hamming distance between them is defined as the minimum number of replacements required to change one of the code strings to the other. For example, the Hamming distance between the binary code strings " 1111 " and " 1001 " is 2 .

The similarity coefficient can describe the degree of similarity between samples. The more similar the two samples, the greater the similarity coefficient. The similarity coefficient $S$ between sample points $X$ and $Y$ needs to meet the following conditions [18]:

$$
\begin{aligned}
& \text { (1) }|S(X, Y)| \leq 1 \\
& \text { (2) }|S(X, Y)|=S(Y, X) \\
& \text { (3) } S(X, X)=1
\end{aligned}
$$

Commonly used similarity coefficients include angle cosine and correlation coefficient, and their calculation formula is as follows [19]:

The angle cosine is

$$
\cos (\theta)=\frac{\sum_{j=1}^{m} x_{1 j} x_{2 j}}{\sqrt{\sum_{j=1}^{m} x_{1 j}^{2}} \sqrt{\sum_{j=1}^{m} x_{2 j}^{2}}} .
$$

When the two vectors are in the same direction, the cosine of the angle is 1 . When they are orthogonal, the cosine of the angle is 0 .

The correlation coefficient is

$$
R(X, Y)=\frac{\sum_{j=1}^{m}\left(x_{1 j}-\overline{x_{1}}\right)\left(x_{2 j}-\overline{x_{2}}\right)}{\sqrt{\sum_{j=1}^{m}\left(x_{1 j}-\overline{x_{1}}\right)^{2}} \sqrt{\sum_{j=1}^{m}\left(x_{2 j}-\overline{x_{2}}\right)^{2}}} .
$$

The correlation coefficient is the cosine of the angle between the standard deviation of the vector, and it indicates the degree of linear correlation between the two vectors.

Information entropy is a quantification method of information, which indicates a measure of the degree of dispersion of distribution. The more scattered the sample distribution, the greater the information entropy; the tighter the sample distribution, the smaller the information entropy. The calculation formula is as follows [20]:

$$
\operatorname{Entropy}(X)=\sum_{i=1}^{n}-p_{i} \log _{2} p_{i}
$$

Among them, $X$ is the sample set, $n$ is the number of sample classifications, and $p$ is the probability of occurrence of the $i$-th type element in $X$.

In people's practice, it is often necessary to deal with data sets with mixed attributes. This kind of data has both numerical and nonnumerical attributes. We have two sample points $X\left(x_{1}, x_{2}, \ldots, x_{m}, \ldots, x_{n}\right)$ and $Y\left(, y_{1}, y_{2}, \ldots, y_{m}, \ldots, y_{n}\right)$. Their first $m$ dimensions are numerical attributes, and $m+1$ to nth are nonnumerical attributes. The distance between them is as follows:

$$
\begin{aligned}
d(X, Y) & =\sqrt{\sum_{i=1}^{m}\left(x_{i}-y_{i}\right)^{2}+\sum_{j=m+1}^{m} \delta\left(x_{k}, y_{k}\right)^{2}}, \\
\delta(a, b) & = \begin{cases}1, & a=b \\
0, & a \neq b\end{cases}
\end{aligned}
$$

In order to solve the clustering problem of nonconvex data sets, people have improved the basic similarity measurement methods, such as manifold distance and kernel distance.

Manifold distance is a new distance measurement method designed for manifold data sets. It can well reflect the similarity between two points in the data set of manifold distribution. It is defined as follows:

(I) The length of the line segment on the manifold: we set $x$ and $y$ as any two points in the space; then, the length of the line segment on the manifold between them is

$$
L\left(x_{i}, x_{j}\right)=\rho^{\operatorname{dist}\left(x_{i}, x_{j}\right)}-1
$$

Among them, dist $\left(x_{i}, y_{j}\right)$ is the Euclidean distance between $x_{i}$ and $y_{j}$, and $\rho<1$ is the expansion factor.

(II) Manifold distance: we regard the data points as the vertices of the graph $G=(V, E)$, and $\rho \in V^{l}$ represents the path connecting the points $p_{1}$ and $p_{|p|}$ of length $l=|p|$ on the graph, where the edge is $\left(p_{k}, p_{k+1}\right) \in E, 1 \leq k \leq P_{|p|} \cdot p_{i j}$ represents the set of all paths connecting the data points $x_{i}$ and $x_{j}$, and the manifold distance is expressed as [21]

$$
D_{i j}=\min \sum_{k=1}^{|p|-1} L\left(p_{k}, p_{k+1}\right) \text {. }
$$

As shown in Figure 1, in the data set of manifold distribution, the similarity of the two points $a$ and $e$ is significantly higher than the similarity of the two points a and $f$, but the Euclidean distance of the former is greater than the latter. If Euclidean distance is used as a measure of similarity, it cannot reflect the spatial consistency of the samples, while the manifold distance can better solve this problem.

Although the manifold distance has obvious advantages in data sets with complex distribution, its computational complexity is higher. Compared with the manifold distance, the core distance has lower computational complexity. The kernel method first maps the original data set to a highdimensional complex nonlinear feature space and then finds the distance between two sample points in this mapping space, which is the kernel distance. In this feature space, the original linearly inseparable data set may become linearly separable. Therefore, it can simplify the structure of the data set and improve the adaptability of the clustering algorithm to complex data sets.

The sample set $\left\{x_{1}, x_{2}, \ldots, x_{N}\right\}$ of the input space is mapped into the feature space and becomes 


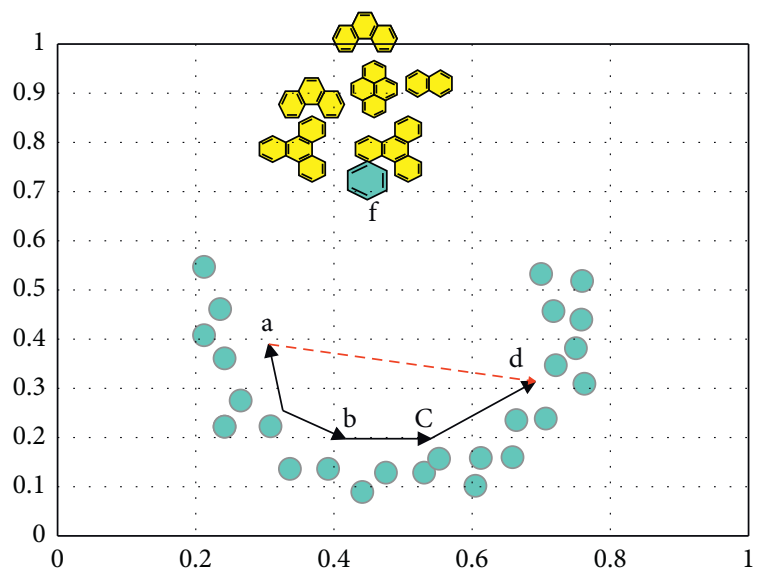

Figure 1: Manifold distance and Euclidean distance.

$\left\{\Phi\left(x_{1}\right), \Phi\left(x_{2}\right), \ldots, \Phi\left(x_{N}\right)\right\}$. Then, the distance (kernel distance) of the sample points $x i, x j$ in the feature space is [22]

$$
\begin{aligned}
d_{H}\left(x_{i}, x_{j}\right) & =\sqrt{\left\|\Phi\left(x_{i}\right)-\Phi\left(x_{j}\right)\right\|^{2}} \\
& =\sqrt{\Phi\left(x_{i}\right) \Phi\left(x_{i}\right)-2 \Phi\left(x_{i}\right) \Phi\left(x_{j}\right) \Phi\left(x_{j}\right) \Phi\left(x_{j}\right)} .
\end{aligned}
$$

It is

$$
d_{H}\left(x_{i}, x_{j}\right)=\sqrt{K\left(x_{i}, x_{i}\right)-2 K\left(x_{i}, x_{j}\right)+K\left(x_{j}, x_{j}\right)} .
$$

Among them, $K(x i, x j)$ is the kernel function, and the commonly used kernel functions are as follows:

(1) Polynomial kernel: $K\left(x_{i}, x_{j}\right)=\left(x_{i} x_{j}+1\right)^{d}$, where $d$ is an integer

(2) Gaussian kernel: $K\left(x_{i}, x_{j}\right)=\exp \left(-\beta\left\|x_{i}-x_{j}\right\|^{2}\right)$, $\beta>0$

(3) Two-layer neural network sigmoidal kernel: $K\left(x_{i}, x_{j}\right)=\tanh \left(-b\left(x_{i} x_{j}\right)-c\right)$, where $b$ and $c$ are custom parameters

As shown in Figure 2, the sample set is linearly inseparable in the original space, but when it is mapped to the feature space through the kernel function, it becomes linearly separable. Because the kernel method is simple and easy to use and can improve the performance of the algorithm, it has been widely used in clustering.

Cluster analysis can divide the original sample set into multiple sample subsets, but is the result of the division reasonable? How to compare the results of different divisions? This requires evaluation of the effectiveness of clustering. A good effectiveness evaluation method can not only judge the performance of the clustering algorithm, but also guide the algorithm to obtain better results. Therefore, the cluster validity evaluation $T$ is also one of the research focuses in the clustering problem.

Criteria for evaluating the pros and cons of clustering algorithms generally include high-dimensionality, scalability, ability to handle noise, discovering clusters of arbitrary shapes, minimizing domain knowledge for determining input parameters, and sensitivity, interpretability, and usability of input record order.

In order to evaluate the clustering results, people have proposed a lot of clustering effectiveness evaluation indicators; these indicators are mainly used to evaluate the tightness between clusters and the degree of dispersion between clusters in the clustering results. There are various clustering effectiveness indicators. The following will focus on the effectiveness indicators that will be used in this paper.

3.1. Clustering Accuracy. The clustering accuracy rate compares the true class labels of each sample with the class labels obtained by clustering and obtains the percentage of correctly divided samples in the sample set. Its definition is as follows:

$$
C C(T, S)=\frac{1}{m} \sum_{i=1}^{T} \max \operatorname{Confusion}(i, j),(i=1, \ldots, T ; j=1, \ldots, S) .
$$

Among them, $m$ represents the number of samples in the sample set to be clustered; $T$ represents the number of true categories of the sample set; $S$ represents the number of categories obtained by clustering; $\operatorname{Confusion}(i, j)$ represents the confusion matrix; in the matrix, $(i, j)$ represents the number of samples that appear in the $i$-th category in the real category and the $j$-th category in the clustered category at the same time. $C C \in[0,1]$, the larger the value, the better the effect of the clustering algorithm.

\subsection{Adjusted Rand Index (ARI) Indicator

$$
R(T, S)=\frac{\left.\sum_{l k}\left(\begin{array}{c}
n_{l k} \\
2
\end{array}\right)-\left[\sum_{l} \begin{array}{c}
n_{n_{l n}} \\
2
\end{array}\right) * \sum_{k}\left(\begin{array}{l}
n_{n k} \\
2
\end{array}\right)\right] /\left(\begin{array}{l}
n \\
2
\end{array}\right)}{(1 / 2)\left[\sum_{l}\left(\begin{array}{c}
n_{l n} \\
2
\end{array}\right)+\sum_{k}\left(\begin{array}{c}
n_{n k} \\
2
\end{array}\right)\right]-\left[\sum_{l}\left(\begin{array}{c}
n_{l n} \\
2
\end{array}\right) * \sum_{k}\left(\begin{array}{l}
n_{n k} \\
2
\end{array}\right)\right] /\left(\begin{array}{c}
n \\
2
\end{array}\right)} .
$$

Among them, nij represents the number of samples belonging to category $I$ and category $k$ at the same time, $k(l$ $e T^{\prime}, k e S$ ), and $T$ and $S$ represent the true category and the category obtained by clustering, respectively. The larger the value, the better the clustering effect. $R(T, S) e[0$, $1]$, the larger the value, the better the effect of the clustering algorithm.

\subsection{Van Dongen (VD) Criterion Index}

$$
V D=\frac{\left(2 N-\sum_{i} \max _{j} n_{i j}-\sum_{j} \max _{i} n_{i j}\right)}{2 N} .
$$

Among them, $n$ represents the number of samples in the sample set, and $n_{i j}$ represents the number of samples of the $i$ th class in the real class and the $j$-th class in the clustered class label at the same time. $V D \in[0,1]$, the smaller the value of $\mathrm{VD}$, the better the clustering effect. 

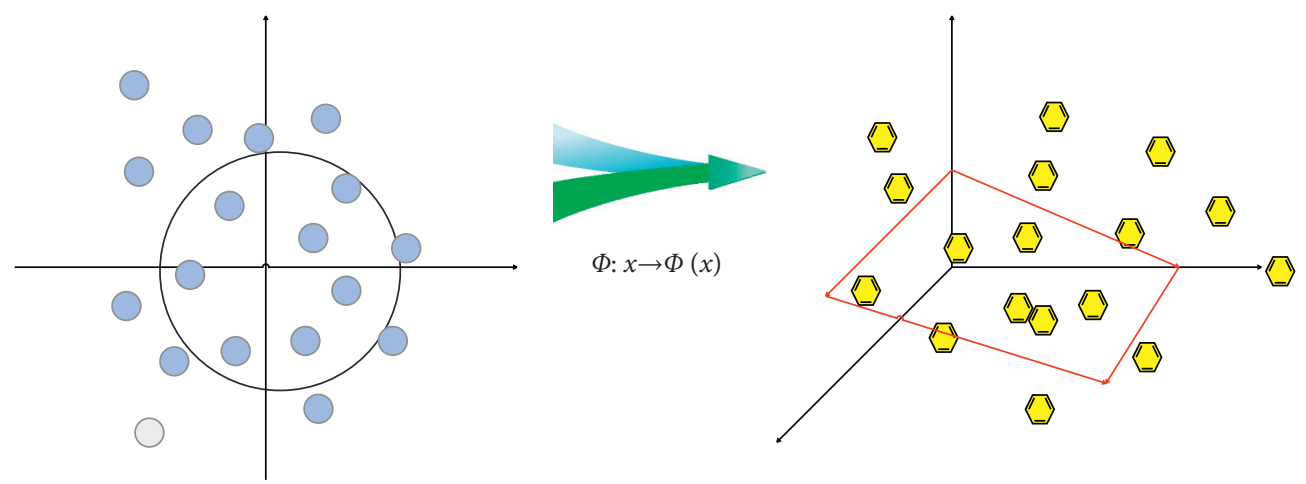

Figure 2: The distribution of samples in the original space and feature space.

\section{IoT-Assisted Hybrid Intelligent Learning Architecture Based on Digital Education in a Diverse Society}

Through the analysis of the system function, after long-term investigation and demonstration, a more feasible design plan is finally determined, as shown in Figure 3:

(1) The system hardware is divided into five parts: main control unit, wireless communication unit, air conditioner infrared remote control unit, lighting control unit, and power supply unit. (2) The main control chip of the main control unit and the lighting control unit adopts STM32F103ZET6, the single-chip microcomputer of the communication unit adopts PIC18F45K22, and the singlechip microcomputer of the air-conditioning infrared remote control unit adopts STM8S103S3P6. The main control unit mainly implements functions such as collection of indoor environment conditions, control of hardware equipment in the classroom, and display of indoor environment. (3) The wireless communication unit mainly realizes the functions of the main control module collecting environmental data and uploading it to the database, sending alarm short messages and receiving control commands. (4) The infrared remote control unit of the air conditioner uses the STM8S103S3P6 chip as the control core, which can realize the functions of learning, storing, restoring, and sending the infrared remote control code of the air conditioner. (6) Indoor environmental quality testing includes testing of information such as temperature and humidity, light intensity, carbon dioxide concentration, formaldehyde content, and PM2.5 concentration. (7) The communication between the hardware device and the server is realized through the SIM900a module. The communication unit uploads the environmental detection information sent by the main control unit to the database via the GPRS network in the POST mode and receives the control information sent by the monitoring platform at the same time. (8) The electric curtains are driven by a decelerating DC motor, the lights are LED energy-saving lamps, and the alarm device uses sound and light and SMS alarm methods. (9) The smart classroom system can judge the distribution of students in the classroom based on the video surveillance images in the classroom, and the light control unit can turn on the lights near the students and adjust the brightness of the lights. The adjustment of the brightness of the light is controlled by the PWM output of the single-chip microcomputer, and the fuzzy control algorithm is adopted to keep the light intensity in the classroom within a proper range.

The whole system needs to realize many functions and cannot be controlled by a single-chip microcomputer. The system is divided into five parts: main control unit, wireless communication unit, air conditioner infrared remote control unit, lighting control unit, and power supply unit. The various parts are relatively independent and cooperate with each other with the main control unit as the core to realize the overall function of the system together. The overall block diagram of the hardware circuit is shown in Figure 4:

The overall hardware structure diagram of the gateway subsystem is shown in Figure 5. A gateway is a network device that serves as the important task of conversion and is used between two systems with different communication protocols and different data formats. Moreover, it can be used for both local area networks and wide area networks.

The software architecture of the gateway subsystem is shown in Figure 6. The lightweight TCP/IP protocol LwIP and WebServer web server run on the FreeRTOS operating system, and the WebServer will call the interface function of LwIP to send and receive data packets to the network. The GCI application that processes the data runs on the WebServer. The data will be sent to the Hyun web page through a form, and finally the data will be sent to the server in the form of the Hunload web page.

According to the main tasks and characteristics of multimedia classroom management, the multimedia classroom integrated management system can be divided into five functional sections. Its structure is shown in Figure 7.

In the design of the application layer of the system, this paper uses the advantages of cloud computing to realize the efficient operation and management of the system and the intelligent interaction of data and information. Moreover, this paper finally transmits the effective data information to the database server of the central control room through the network and displays it on the electronic screen. The system can remotely monitor and control the terminal teaching equipment of the multimedia classroom in real time, can accurately express the status information of various equipment in the classroom and the cause of the failure, and 


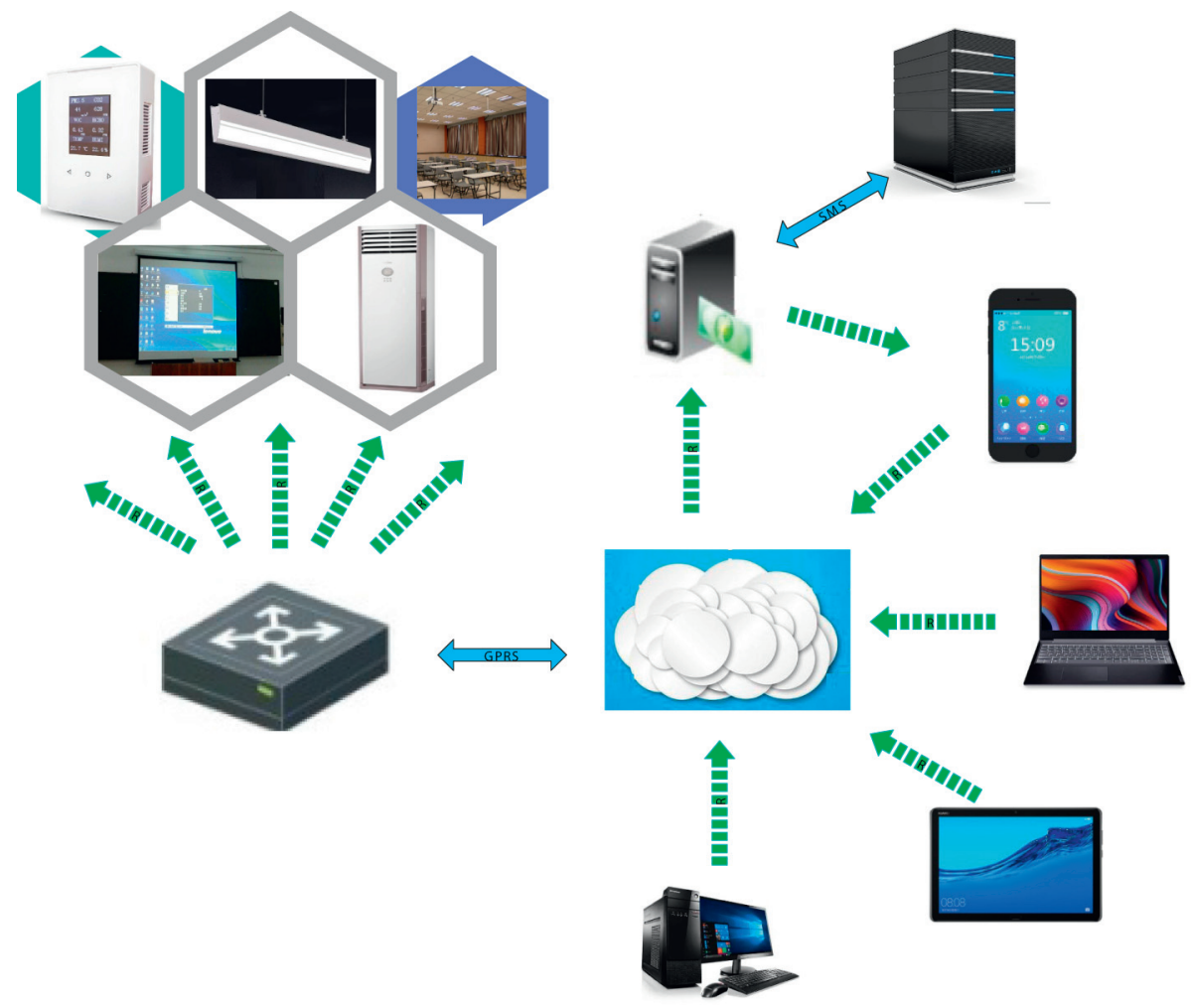

FIGURE 3: Block diagram of a smart classroom system based on Internet of Things technology.

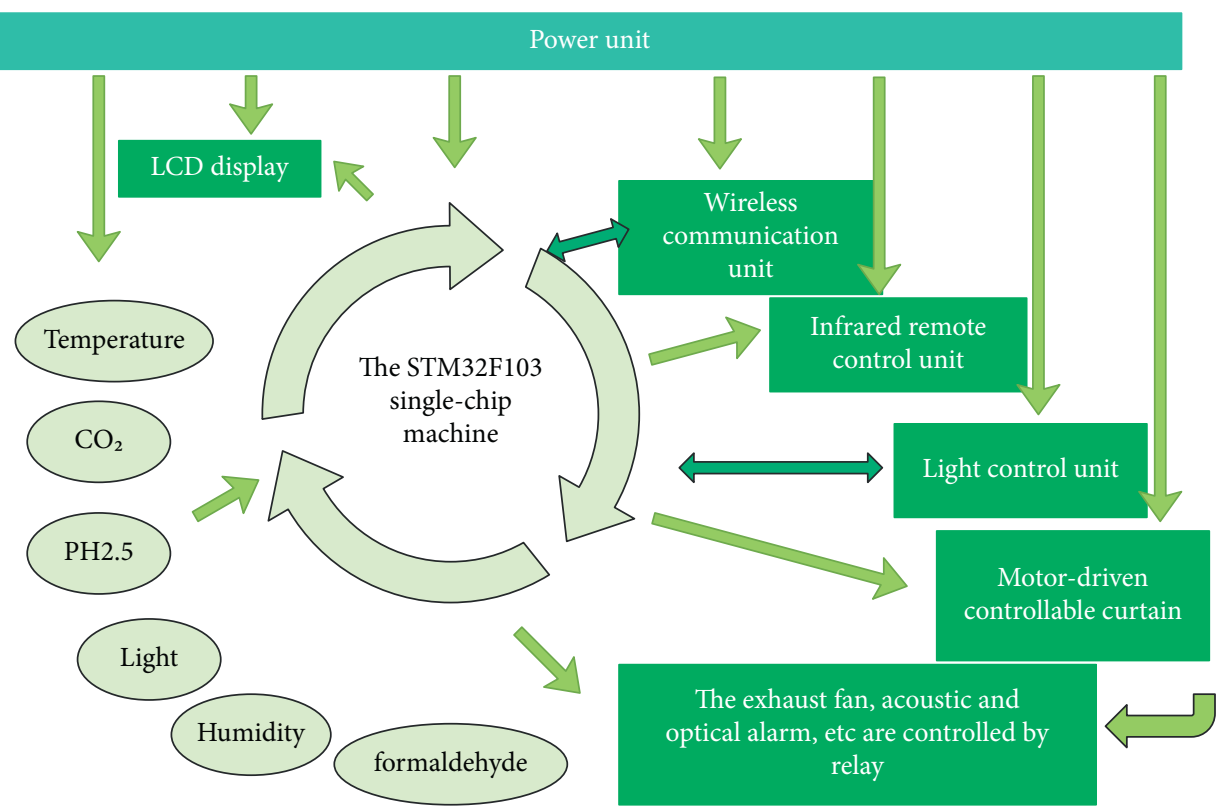

FIgURE 4: Overall block diagram of the hardware circuit. 


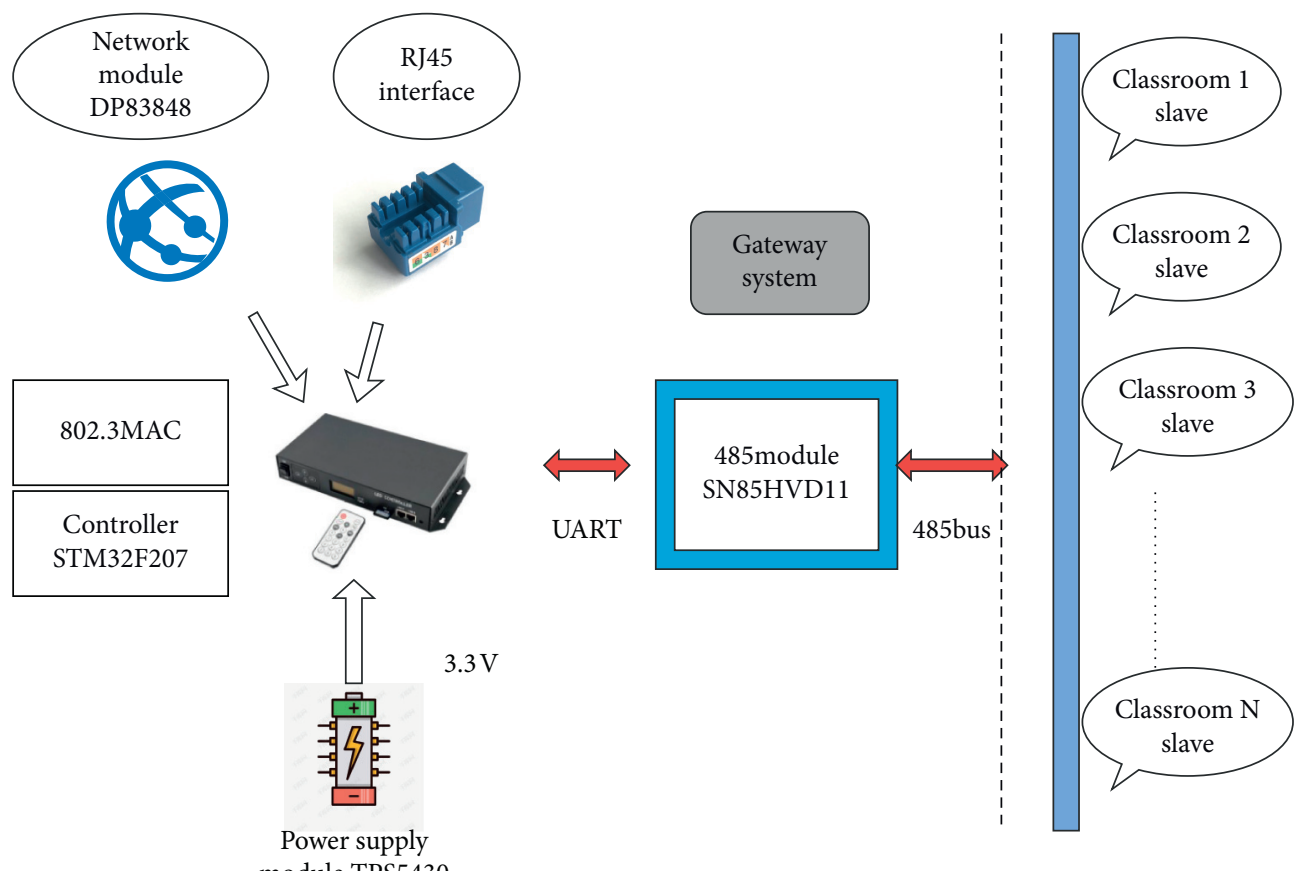

FIGURE 5: Block diagram of the overall hardware structure of the gateway subsystem.

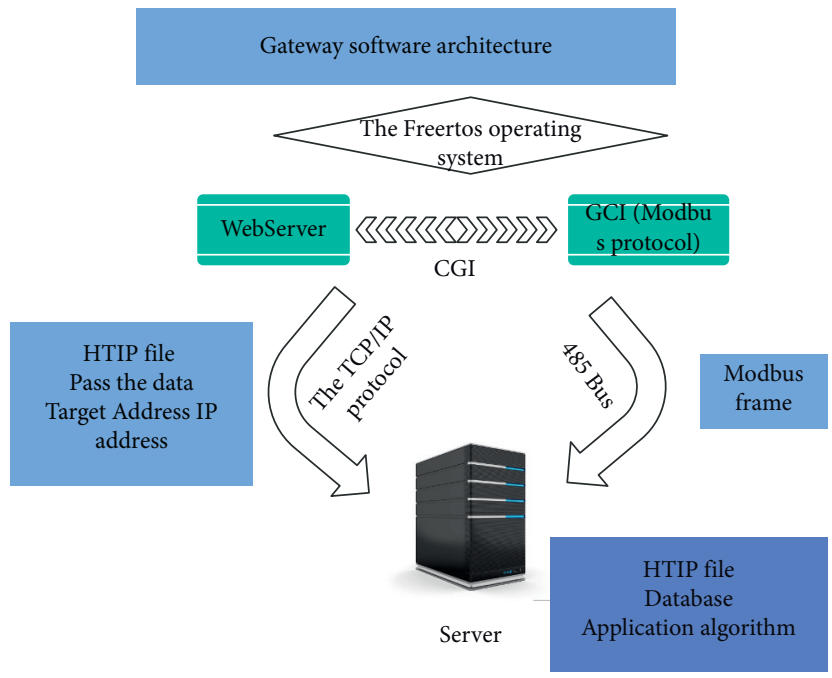

Figure 6: The software architecture of the gateway subsystem.

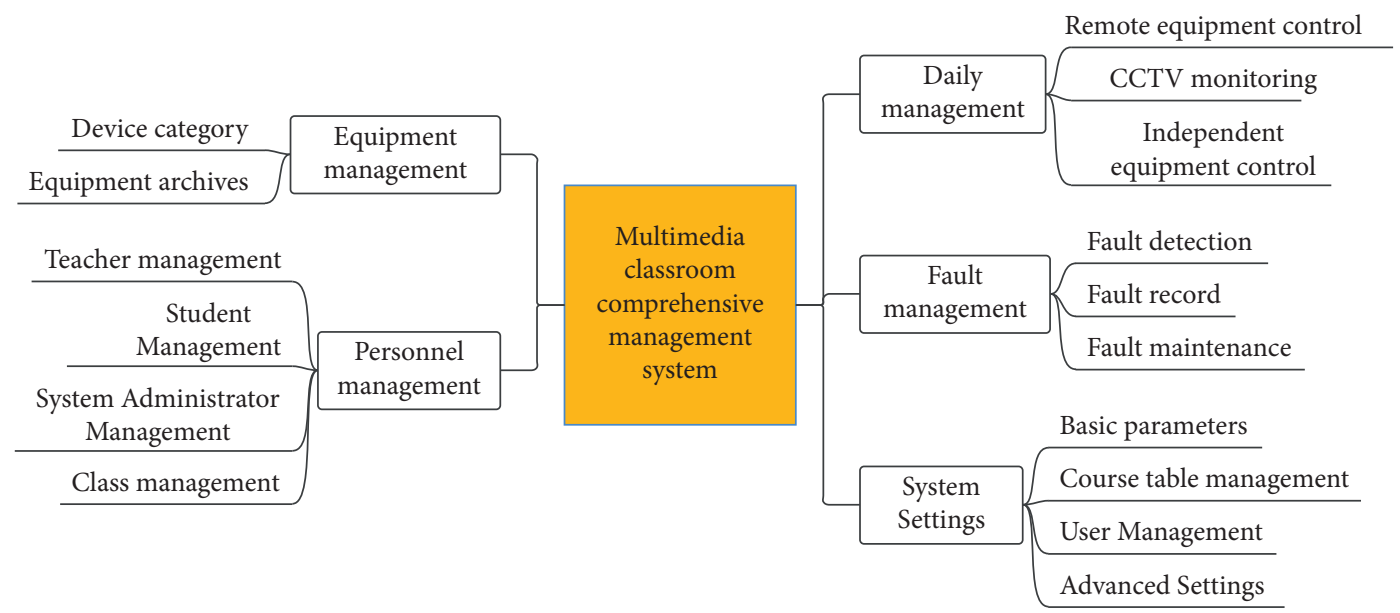

FIgURE 7: Multimedia classroom integrated management system based on the Internet of Things. 


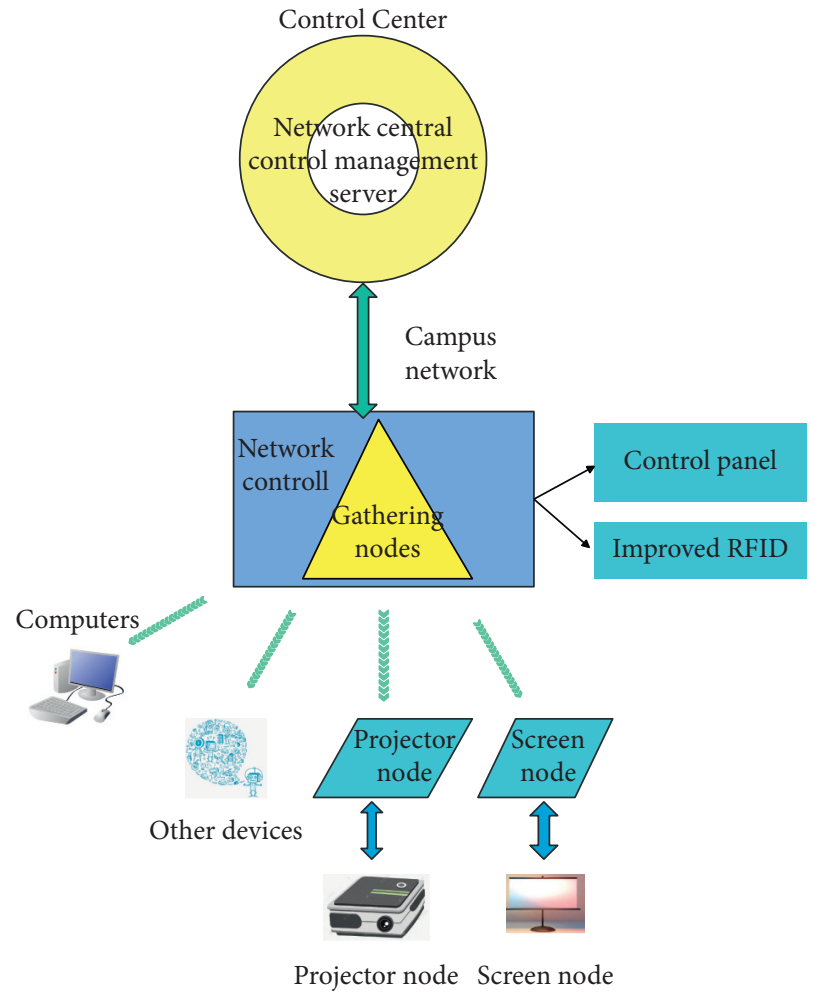

FIGURE 8: Network topology of multimedia classrooms based on the Internet of Things.

TABLe 1: System experimental performance.

\begin{tabular}{|c|c|c|c|c|c|}
\hline Number & Digitizing & Teaching effect & Number & Digitizing & Teaching effect \\
\hline 1 & 94.6 & 86.6 & 25 & 87.3 & 89.9 \\
\hline 2 & 96.1 & 79.5 & 26 & 91.6 & 86.9 \\
\hline 3 & 96.1 & 81.5 & 27 & 89.7 & 90.5 \\
\hline 4 & 96.1 & 80.5 & 28 & 91.2 & 90.2 \\
\hline 5 & 87.3 & 80.4 & 29 & 91.5 & 91.6 \\
\hline 6 & 90.7 & 79.3 & 30 & 96.9 & 88.3 \\
\hline 7 & 93.7 & 88.0 & 31 & 92.8 & 88.4 \\
\hline 8 & 89.7 & 88.2 & 32 & 89.9 & 86.7 \\
\hline 9 & 93.3 & 82.7 & 33 & 93.5 & 80.7 \\
\hline 10 & 96.4 & 88.7 & 34 & 95.6 & 93.1 \\
\hline 11 & 94.9 & 86.8 & 35 & 88.0 & 87.0 \\
\hline 12 & 90.9 & 84.0 & 36 & 92.1 & 87.7 \\
\hline 13 & 90.8 & 89.0 & 37 & 92.5 & 89.7 \\
\hline 14 & 90.5 & 82.1 & 38 & 92.1 & 79.7 \\
\hline 15 & 94.1 & 93.2 & 39 & 87.1 & 89.0 \\
\hline 16 & 92.1 & 90.7 & 40 & 96.5 & 80.4 \\
\hline 17 & 92.1 & 80.9 & 41 & 92.4 & 85.7 \\
\hline 18 & 88.6 & 83.5 & 42 & 96.6 & 89.9 \\
\hline 19 & 88.2 & 88.6 & 43 & 88.3 & 93.5 \\
\hline 20 & 92.0 & 82.2 & 44 & 88.2 & 93.4 \\
\hline 21 & 87.0 & 85.7 & 45 & 91.5 & 81.9 \\
\hline 22 & 87.4 & 80.8 & 46 & 96.4 & 92.6 \\
\hline 23 & 94.4 & 83.7 & 47 & 89.9 & 85.6 \\
\hline 24 & 96.0 & 93.6 & 48 & 90.8 & 81.7 \\
\hline
\end{tabular}

can also assist the administrator to solve the equipment failure remotely and improve work efficiency. In addition, it can help teachers in class operate and use various teaching equipment correctly. The network topology of the system is shown in Figure 8.
This paper verifies the performance of the above-built system. Starting from the actual situation of intelligent education, this paper combines the simulation system to build an intelligent IoT-assisted hybrid intelligent learning architecture. Moreover, this paper evaluates the teaching 


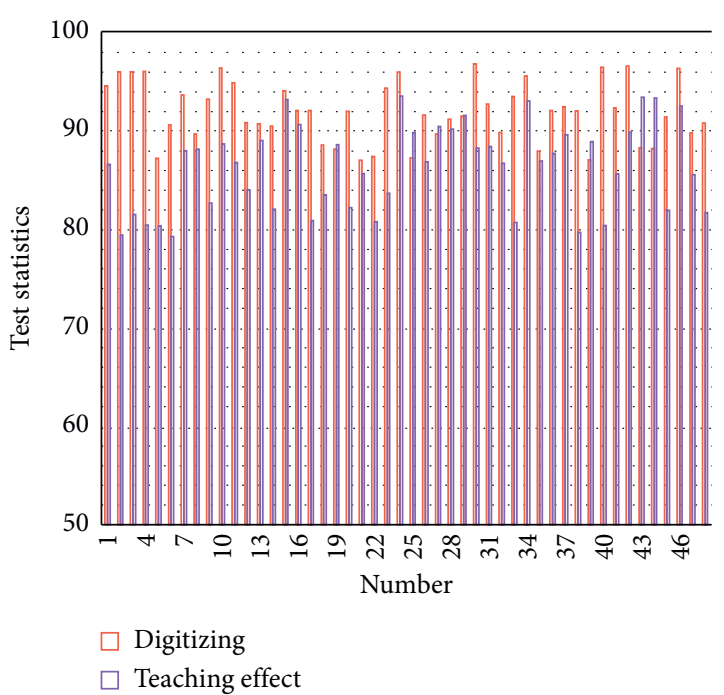

Figure 9: Performance statistics of IoT-assisted hybrid intelligent learning architecture based on digital education in a diverse society.

effect of the system from the digitization of the educational resources of the intelligent learning system and the teaching effect, and the results shown in Table 1 and Figure 9 are obtained.

From the above research, it can be seen that the IoTassisted hybrid intelligent learning framework based on digital education in a diverse society has a certain occasional effect, and it can be used as a reference for the development of subsequent intelligent education.

\section{Conclusion}

This article introduces the elements of the Internet of Things into the digital intelligent education system. On the one hand, this paper builds an IoT smart laboratory to provide technical and environmental support for digital intelligent education experiments and remote sharing of equipment resources. On the other hand, this paper constructs the Internet of Things digital intelligent education with the concept and technical characteristics of the Internet of Things, which makes the digital intelligent education smarter, more interactive with the outside world, and more concrete and vivid experimental results. The digital intelligent education practice teaching system that incorporates the Internet of Things technology is also in line with the theoretical requirements of the situational teaching, task-driven teaching, and research teaching methods advocated by higher education. Moreover, it conforms to the cognitive and psychological characteristics of college students' intense pursuit of new knowledge and new things and conforms to the goals and directions of quality education and innovation education in contemporary universities. This article combines the Internet of Things technology to construct a hybrid intelligent learning system, applies it to digital education in a diverse society, and validates the system to provide a theoretical reference for subsequent intelligent education.

\section{Data Availability}

The labeled dataset used to support the findings of this study is available from the corresponding author upon request.

\section{Conflicts of Interest}

The author declares no competing interests.

\section{Acknowledgments}

This study was sponsored by first-class undergraduate courses in Henan Province: Modern Educational Technology and Application; School-level Excellent Teaching Grassroots Organization of Zhengzhou Normal University (JCZZ-20150).

\section{References}

[1] S. Liu, P. Chen, and P. Chen, "Research on fuzzy comprehensive evaluation in practice teaching assessment of computer majors," International Journal of Modern Education and Computer Science, vol. 7, no. 11, pp. 12-19, 2015.

[2] L. Zhou, H. Li, and K. Sun, "Teaching performance evaluation by means of a hierarchical multifactorial evaluation model based on type-2 fuzzy sets," Applied Intelligence, vol. 46, no. 1, pp. 1-11, 2016.

[3] J. Porozovs, L. Liepniece, and D. Voita, "Evaluation of the teaching methods used in secondary school biology lessons," Journal of Pedagogy and Psychology "Signum Temporis", vol. 7, no. 1, pp. 60-66, 2015.

[4] M. A. Oliveros, A. García, and B. Valdez, "Evaluation of a teaching sequence regarding science, technology and society values in higher education," Creative Education, vol. 6, no. 16, pp. 1768-1775, 2015.

[5] M. Sánchez Cerón and F. M. d. S. Corte Cruz, "The evaluation of teaching: some consequences for Latin America," Revista Mexicana de Investigación Educativa, vol. 20, no. 67, pp. 1233-1253, 2015.

[6] K. Angell and E. Tewell, "Teaching and un-teaching source evaluation: questioning authority in information literacy instruction," Comminfolit, vol. 11, no. 1, pp. 95-121, 2017.

[7] M. Brkovic and P. Chiles, "'Spector-the sustainability inspector": participatory teaching, learning and evaluation game for architects, architecture students and pupils," Facta Universitatis-Series: Architecture and Civil Engineering, vol. 14, no. 1, pp. 1-20, 2016.

[8] Y. Jiang and Y. Wang, "Evaluation of teaching quality of public physical education in colleges based on the fuzzy evaluation theory," Journal of Computational and Theoretical Nanoscience, vol. 13, no. 12, pp. 9848-9851, 2016.

[9] K. Royal, "A guide for making valid interpretations of student evaluation of teaching (SET) results," Journal of Veterinary Medical Education, vol. 44, no. 2, pp. 1-7, 2016.

[10] F. Garofalo, P. Mota-Moya, A. Munday, and S. Romy, “Total extraperitoneal hernia repair: residency teaching program and outcome evaluation," World Journal of Surgery, vol. 41, no. 1, pp. 1-6, 2017.

[11] G. Costa and R. Ortale, "Model-based collaborative personalized recommendation on signed social rating networks," ACM Transactions on Internet Technology, vol. 16, no. 3, pp. 1-21, 2016. 
[12] Y. Guan, Q. Wei, and G. Chen, "Deep learning based personalized recommendation with multi-view information integration," Decision Support Systems, vol. 118, pp. 58-69, 2019.

[13] C. Yang, Q. Fan, T. Wang et al., "RepoLike: a multi-featurebased personalized recommendation approach for opensource repositories," Frontiers of Information Technology \& Electronic Engineering, vol. 20, no. 2, pp. 86-101, 2019.

[14] Q. Lin, X. Wang, B. Hu et al., "Multiobjective personalized recommendation algorithm using extreme point guided evolutionary computation," Complexity, vol. 2018, Article ID 1716352, 18 pages, 2018.

[15] X. U. Dazhi, "Research on music culture personalized recommendation based on factor decomposition machine," Personal and Ubiquitous Computing, vol. 24, no. 2, pp. 247257, 2020.

[16] N. X. Bach, N. D. Hai, and T. M. Phuong, "Personalized recommendation of stories for commenting in forum-based social media," Information Sciences, vol. 352-353, no. 2, pp. 48-60, 2016.

[17] D. Guo, Y. Zhu, W. Xu, S. Shang, and Z. Ding, "How to find appropriate automobile exhibition halls: towards a personalized recommendation service for auto show," Neurocomputing, vol. 213, pp. 95-101, 2016.

[18] C. Guilin, Z. Xuzhen, Y. Zhao, and H. Tian, "Personalized recommendation via suppressing excessive diffusion," Mathematical Problems in Engineering, vol. 2017, Article ID 2587069, 10 pages, 2017.

[19] U. Liji, Y. Chai, and J. Chen, "Improved personalized recommendation based on user attributes clustering and score matrix filling," Computer Standards \& Interfaces, vol. 57, no. 5, pp. 59-67, 2018.

[20] T. Ha and S. Lee, "Item-network-based collaborative filtering: a personalized recommendation method based on a user's item network," Information Processing \& Management, vol. 53, no. 5, pp. 1171-1184, 2017.

[21] W. Intayoad, T. Becker, and P. Temdee, "Social context-aware recommendation for personalized online learning," Wireless Personal Communications, vol. 97, no. 1, pp. 1-17, 2017.

[22] J. Xia, G. Li, and Z. Cao, "Personalized exercise recommendation algorithm combining learning objective and assignment feedback," Journal of Intelligent and Fuzzy Systems, vol. 35, no. 3, pp. 2965-2973, 2018. 